\title{
TOP-UP OPERATION EXPERIENCE AT THE ADVANCED PHOTON SOURCE *
}

\author{
L. Emery, M. Borland, ANL, Argonne, IL
}

\begin{abstract}
The Advanced Photon Source (APS) is a 7-GeV, thirdgeneration synchrotron radiation source. To provide more stable beam for users, in September 1998 we began commissioning a new operating mode called "top-up." In this mode, the beam current does not decay but is maintained at a high level using frequent injection, while photon shutters are open and photon beams are delivered to users. The hardware, software, and safety requirements for top-up will be reported. Safety issues related to injection with open photon shutters are covered in companion papers in this conference. Present operational experience includes testing aspects of top-up injection and delivering beam to Xray users for a few hours with fractional current stability of $10^{-3}$. We expect to run several top-up operation shifts in Spring 1999. Issues of importance are orbit and emittance transients during the injection and scheduling of injection pulses for the convenience of users.
\end{abstract}

\section{INTRODUCTION}

Top-up injection refers to injecting with photon shutters open to deliver a near-constant stored beam current. This will improve X-ray beam stability through a constant heat load on X-ray optics and eliminate current-dependent systematics of storage ring (SR) beam diagnostics.

A relative current stability of $10^{-4}$ is our long-term goal. We have acheived this for a few hours of running during machine studies (shutters not necessarily open). A lower stability of $10^{-3}$ was achieved routinely, and has been delivered to users for several hours on a trial basis.

An equally important beam quality issue of top-up is the beam disturbance that may be caused by the injection process. Though any closed orbit or emittance disturbance is damped out after several tens of milliseconds through synchrotron radiation damping or the decay of pulsed magnet fields, these disturbances affect most X-ray experiments. We will report on steps taken to reduce the impact of these injection transients on X-ray experiments.

\section{RADIATION SAFETY}

Photon shutters are normally closed during injection to block any injected beam particles from escaping the SR enclosure and entering the experiment hall, where they would constitute a radiation hazard. (This might occur, say, due to a short in a dipole magnet with a photon port.) Prevention of such an accident is the main safety issue in top-up and was the subject of extensive studies $[1,2]$.

\footnotetext{
${ }^{*}$ Work supported by U.S. Department of Energy, Office of Basic Energy Sciences, under Contract No. W-31-109-ENG-38.
}

We found that if the dipole magnets and power supplies are operating normally, no configuration error or magnet fault can produce an accident. Simulations gave a stronger conclusion, that even with a shorted dipole and other errors, one cannot have stored beam while extracting injected beam down a photon beamline. Thus, we ensure the top-up safety with an interlock that inhibits injection with shutters open if there is no stored beam.

The simulations used a series of lattices and conservative (i.e., accident-enhancing) choices of magnet faults. All scenarios involved a shorted dipole of variable degree. Other faults considered simultaneously were hypothetical mis-set quadrupoles, worst-case dipole steering from malfunctioning nearby multipoles, and injected beam energy error. To limit the possible steering from multipoles, we ran the simulations for $6 \mathrm{GeV}$; a hardware interlock on the dipole power supply enforced this minimum energy.

Because of the importance of aperture location and dimensions in limiting the possible trajectories of the injected beam, we require controlled drawings and documents listing the relevant apertures (those used in the tracking simulations). Tolerances for the placement of apertures (determined from tracking) are also documented. Routine checks of the placement of apertures are required. Since apertures in the SR vacuum chambers and photon beamline are not visible from the outside, this is done indirectly using survey measurements of the magnets and photon beamline safety shutter, and using measurement gauges to verify the position of vacuum chambers in magnets.

\section{OTHER OPERATIONAL CONCERNS}

The injector produces a single bunch at $2 \mathrm{~Hz}$, with a nominal charge of $1 \mathrm{nC}$ and design maximum of $20 \mathrm{nC}$. For $10^{-4}$ current stability, the injected charge is about $0.04 \mathrm{nC}$, at the bottom range of the beam transport line diagnostics sensitivity. Beam diagnostics with higher sensitivity are planned. In the meantime, scrapers in the transport line can be used to scrape down to the required low charge.

Permanent magnets in insertion devices (IDs) can be demagnetized by a large radiation dose. Injected beam losses are highest at the ID vacuum chambers (VCs). In top-up operation the IDs are closed, almost touching the VCs. Shielding cannot reduce the dose at the downstream end of the ID since a radiation shower travels inside the beam pipe and through the thin $(1 \mathrm{~mm}) \mathrm{Al}$ chamber. Injection losses will have to be closely monitored. We plan to install radiation monitors on the ID VC to serve as diagnostics.

SR injection uses a four-magnet kicker bump lasting less than one turn, as well as two septa at the end of the transfer line. Poor injection efficiency is typically due to variation 
of injected beam initial coordinates in the SR that causes particles to hit vertical or horizontal apertures, which are both small because of small gap undulator apertures. Automatic trajectory correction takes care of the vertical and most of the horizontal motion. However, horizontally we are affected by the pulse-to-pulse jitter of the pulsed magnets. Improved pulsed supply performance and increased injection aperture in the final septum are planned for the near future. In the meantime, virtually $100 \%$ injection efficiency can be obtained using a mismatched kicker bump that reapportions the betatron oscillation of the injected beam between the injected beam and the stored beam.

\section{BEAM PERTURBATION}

It is important that the injection process is transparent to the users. Stored beam centroid motion or increased emittance will reduce the $\mathrm{X}$-ray brightness. The mismatched injection bump used for good injection perturbs the stored beam, imparting a betatron amplitude of a few millimeters. Decoherence (due to tune spread) occurs in about 20 turns, replacing the centroid motion with increased emittance. An increase in measured beamsize (Figure 1) is seen after injection and lasts a few damping times, during which time the synchrotron radiation brightness is greatly reduced. The beam returns to normal in about $30 \mathrm{~ms}$. The blow-up is expected to be greatly reduced when aperture problems are fixed, which will allow a matched kicker bump.

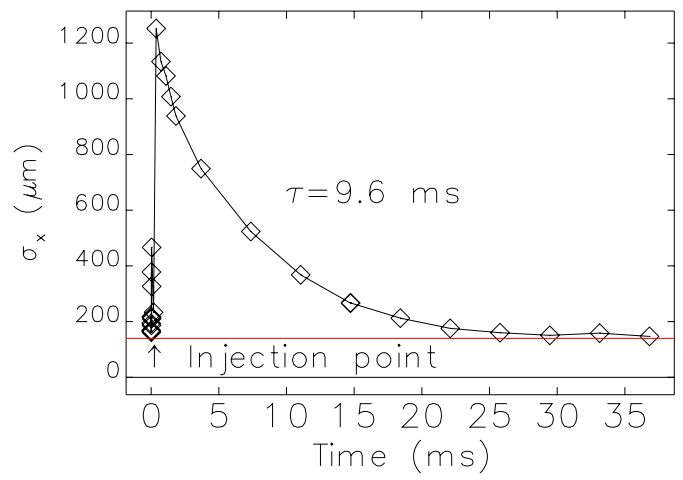

Figure 1: Beamsize blow-up after firing injection kickers

Another injection-related beam perturbation is a closed orbit distortion produced by a septum leakage field. The first, "thick" septum, pulsed with a $10 \mathrm{~ms}$ half sine wave, almost touches the SR vacuum chamber. Figure 2 shows the orbit disortion caused by the leakage field for the original septum and an upgraded septum. In either case the beam disturbance lasts for a total of about $25 \mathrm{~ms}$. The maximum orbit distortion for the upgraded septum is about $200 \mu \mathrm{m}$-about $60 \%$ of the horizontal beam size. Various beamlines will experience different orbit and angle distortion depending on the phase advance from the septum.

The remaining closed orbit distortion will be corrected using the real-time orbit feedback system in feedforward mode in a time window around the injection transient. While this system cannot react quickly enough to com-

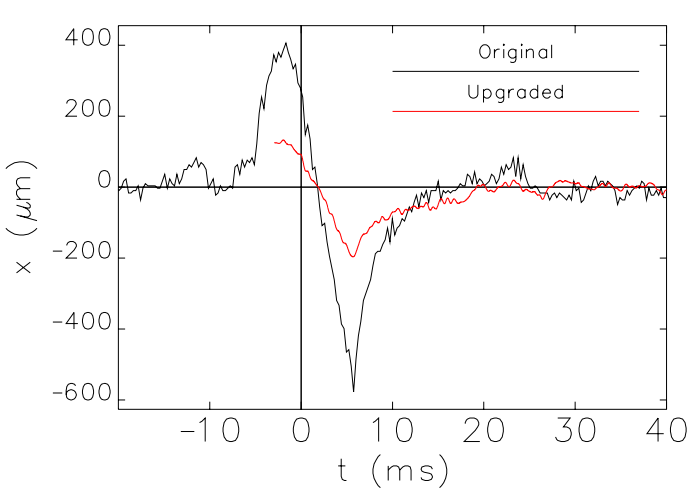

Figure 2: Closed orbit bump due to septum leakage

pletely eliminate the distortion in feedback mode, in feedforward mode its $1.6-\mathrm{kHz}$ update rate should suffice.

Performance of real-time feedback was affected indirectly by the injection orbit transient because of the strong coupling in the mini-BPMs between the beam size and the $y$-readback for an off-centered beam in the $y$ direction. The problem is eliminated by gating off the feedback for the duration of the injection transient.

Injection-related trigger signals are available to X-ray experimenters so that $\mathrm{X}$-ray data acquisition can be blocked during a time window around the injection event. This is most useful for counting experiments. The possible blanking out of $35 \mathrm{~ms}$ of beam time every two minutes is not problematic, as it provides a $99.97 \%$ duty factor.

Those experimenters taking data by CCD camera imaging require uninterrupted beam for a wide range of time intervals, say 1 second to 30 minutes. Any beam disturbance that reduces X-ray brightness during a long CCD camera scan may produce an unacceptable spurious signal. Since our current injection process produces a beam perturbation, in the short term we must adopt a top-up injection time interval longer than the longest CCD camera experiment, say one to several hours following a strict schedule. This mode does not provide all the advantages of top-up, but can significantly increase X-ray availability.

\section{COMMISSIONING}

Commissioning of top-up operation mode involves injecting beam in the SR with the photon shutters open for the first time. The commissioning plan entails measuring radiation dose rates outside shielding walls in areas possibly occupied by personnel for various beam loss scenarios and checking that the measured doses are within the acceptable predicted values. The beam loss scenarios considered here are those that are possible assuming the proper functioning of the personnel protection system. The first scenario is an injected beam loss inside the SR enclosure; the second, an injected beam missteered at an ID vacuum chamber.

The maximum radiation hazard is expected to be produced when the injected beam is missteered into an ID vacuum chamber aperture. Bremstrahlung photons travel through the open shutters and scatter in the front-end en- 
closure (FOE), producing a dose outside the FOE. Measurements we done simulating this condition by repeatedly dumping a stored beam on different ID VCs with the corresponding beamline shutters open. The worst results were scaled to the injection safety envelope of $40 \mathrm{nC} / \mathrm{s}$, giving a maximum credible incident of $12.5 \mathrm{rem} / \mathrm{h}$.

The above measurement was repeated with an actual 1$\mathrm{nC} / \mathrm{s}$ injected beam dumped on two separate ID vacuum chambers with corresponding beamline shutters open. The largest dose measured around the FOE scaled to the safety envelope conditions was $0.25 \mathrm{rem} / \mathrm{h}$. The variability of the equipment housed in the FOEs may explain the discrepancy between these two experiments.

Assuming a 100-mA beam with 10 hour lifetime, a current stability of $10^{-3}$ requires injection of $0.37 \mathrm{nC}$ every 36 seconds. The maximum dose rate outside the SR tunnel if every pulse was lost on an ID VC would be $3.2 \mathrm{mrem} / \mathrm{h}$.

The tracking studies of top-up safety cannot be verified easily as it would involve defeating the personnel protection system and creating difficult-to-generate magnet faults. However, we verified one aspect of the simulations. The tracking studies give a conservative limit for the negative fractional strength error (FSE) for a dipole magnet that would preclude stored beam. The tracking limit, FSE $=-0.09$, would be supported if the measured limit was smaller in absolute value. Dipole trim coils were used to simulate a negative FSE in a particular dipole. Beam was lost at FSE $=-0.050(-0.036)$ with (without) orbit correction running.

\section{TOP-UP OPERATION}

There are two top-up operation modes, a high currentstability mode (frequent injection) and a refill mode (injection every few hours). The relative current stability, $\epsilon$, is defined by $\left|I(t)-I_{0}\right|<\epsilon I_{0}$ where $I(t)$ is the instantaneous current and $I_{0}$ is the target current.

In high current-stability mode we either inject on an asneeded basis or at a constant time interval. For the former, injection occurs when the beam decays below $I_{0}$. Because of typical $10 \%$ injector performance variation, injection occurs at irregular intervals. Injecting with 0.04-nC charge, we achieved $10^{-4}$ stability for $I_{0}=100 \mathrm{~mA}$, with an injection interval of about $7 \mathrm{~s}$. A $3 \times 10^{-4}$ current stability was achieved by injecting $1 \mathrm{nC}$ every few minutes.

Since the unpredictability of the injection event in this mode is undesirable to users who are sensitive to the injection process, we developed a fixed-injection-interval mode. One specifies an injection interval $T$, a target current $I_{0}$, and set up the injector to deliver the expected charge deficit $\left(\Delta I=I_{0} T / \tau\right)$ in one pulse. At the end of the interval, we inject only if $I<I_{0}$, giving stability of $2 \Delta I$.

In the refill top-up mode the beam is allowed to decay undisturbed for several hours, then beam is injected to reach the target current. For example, in one hour the $100-\mathrm{mA}$ beam decays by 3 to $5 \mathrm{~mA}$, depending on the bunch pattern. Refilling at $2 \mathrm{~Hz}$ and $0.8 \mathrm{nC} /$ pulse would take 8 to 12 seconds, giving a high duty factor $(99.7 \%)$ and a low user impact. Presently standard APS operation involves filling (with shutters closed) to $100 \mathrm{~mA}$ every 12 or 24 hours. We plan to change this to refill-mode top-up to improve X-ray availability and get more experience with top-up injection.

Obviously when the injector becomes unavailable because of some fault condition, then the beam current in the high current-stability mode will decay below the current tolerance. When the injector is ready again, the current will be topped-up to the target current with a sequence of pulses, similar to what happens in refill mode.

In top-up injection with high current-stability, only one pulse in one bucket is injected. In general, a different target bucket is selected at every pulse to maintain the bunch pattern as well as the total current. A constant bunch pattern is desirable for BPM triggering and electrical offset stability, and for X-ray user timing experiments.

The target bucket is selected between top-up injection events, during which time the bunch pattern intensity is measured, averaged, and compared with a reference bunch pattern intensity. The bunch that has the largest charge deficit will receive the charge of the next injection. The stability of the bunch charge is of the order of $\epsilon$ times the number of bunches. For example, for a standard bunch pattern of about 80 bunches, the bunch current variation could be as high as $8 \%$.

We measured the jitter in the timing of the injection pulses in response to user concerns of possible mistimed injection pulses. We found no missed or skipped injection pulses in several hours of testing, and measured the jitter in time to be $60 \mathrm{~ms}$ in a one-hour measurement [3]. This jitter is consistent with the $60-\mathrm{Hz}$ line frequency drift.

We plan to implement pulse histories for the SR pulsed magnets, particularly in the kickers, in order to help diagnose possible beam dumps during top-up. Though the kickers have been very reliable, and we have no evidence of kicker-caused beam dumps in the past, it would be useful to be able to eliminate this possibility when diagnosing causes of beam dumps.

\section{ACKNOWLEDGEMENTS}

The authors wish to acknowledge the work of all the techical groups at APS for making top-up injection possible. Thanks to B. Yang for supplying the data for Figure 1.

\section{REFERENCES}

[1] L. Emery, M. Borland, "Analytical Studies of Top-Up Safety at the Advanced Photon Source," these proceedings.

[2] M. Borland, L. Emery, "Tracking Studies of Top-Up Safety at the Advanced Photon Source,", these proceedings.

[3] F. Lenkszus, R. Laird, private communication. 